\title{
DYNAMIC BUCKLING OF A DAMPED IMPERFECT COLUMN ON A NONLINEAR FOUNDATION*
}

By

\author{
DEBORAH F. LOCKHART
}

Michigan Technological University, Houghton

\begin{abstract}
The dynamic buckling of a finite damped imperfect column which rests on a nonlinear foundation and which is subjected to axial step-loading is studied using a perturbation method. The imperfection is considered to be small. A simple asymptotic expression for the dynamic buckling load is obtained in terms of the Fourier component of the imperfection in the shape of the classical buckling mode. This leads to simple relations, independent of the imperfection, between the dynamic and static buckling loads for the damped and undamped column.
\end{abstract}

Introduction. It has been shown that large reductions in the buckling strengths of certain structures are caused by the presence of small geometrical and physical imperfections. These structures are called "imperfection-sensitive". Koiter [1,2] formulated a static theory of post-buckling behavior which was extended to dynamic buckling by Budiansky and Hutchinson [3, 4, 5]. They assumed that the imperfections were in the shape of the classical buckling mode. In [6] it was shown that this restriction need not be imposed. In this paper a damped finite column with a small modal imperfection is studied. The column rests on a nonlinear elastic foundation and is subjected to step-loading. The static buckling of this structure has been studied in [7, 8,9] and the dynamic buckling of the undamped column in [6]. A formal multi-scale perturbation expansion will be used to study the column. In [10] such a method was used to study the dynamic response of an imperfection-sensitive structure.

Differential equation. We consider a damped finite column which is laterally supported by a continuous elastic foundation. The column is subjected to an axial load which is suddenly applied and subsequently kept at a constant value. The nondimensional form of the equation governing the lateral displacement $w(x, t)$ of the column is

$$
\begin{gathered}
w_{t t}+\gamma w_{t}+w_{x x x x}+2 \lambda w_{x x}+w-\alpha w^{3}=-2 \lambda \epsilon \bar{w}_{x x}, \quad 0<x<\pi, t>0 \\
w=w_{x x}=0 \text { at } x=0, \pi ; t>0 \\
w(x, 0)=\epsilon f(x), w_{t}(x, 0)=\epsilon g(x), \quad 0<x<\pi
\end{gathered}
$$

where a subscript in $x$ or $t$ denotes partial differentiation with respect to that variable and $\alpha=1$ or $\alpha=-1$ depending on whether the foundation acts like a "softening" or "hardening" spring. The nondimensional axial coordinate $x$, lateral displacement $w$, time $t$, axial load parameter $\lambda$, damping coefficient $\gamma$, and stress-free initial displacement $\bar{w}$ (the

\footnotetext{
* Received March 17, 1977; revised version received August 9, 1977.
} 
imperfection) are related to the corresponding physical quantities by

$$
\begin{array}{ccc}
x=\left(k_{1} / E I\right)^{1 / 4} X, & w=\left(k_{3} / k_{1}\right)^{1 / 2} W, \quad t=\left(k_{1} / m\right)^{1 / 2} T, \\
\lambda=P / 2\left(E I k_{1}\right)^{1 / 2}, & \gamma=Q /\left(m k_{1}\right)^{1 / 2}, \quad \epsilon \bar{W}=\left(k_{3} / k_{1}\right)^{1 / 2} \bar{W} .
\end{array}
$$

Nonlinear geometric effects and axial inertia are neglected. It is assumed that the nondimensional initial displacements and velocities are of the same order as the imperfection ( $\epsilon$ is a small parameter); if the initial conditions are of an order lower than the imperfection, then the results of the analysis must be modified. $E I$ is the bending stiffness of the column, $P$ is the magnitude of the axial step-loading applied at $T=0$ and $m$ is the mass per unit length of the column. A foundation that produces a restoring force per unit length of $k_{1} W-\alpha k_{3} W^{3}$ restrains the column against lateral deflection $W$.

The classical problem (linear, time-independent and $\bar{w} \equiv 0$ ) consists of

$$
\begin{gathered}
w_{x x x x}+2 \lambda w_{x x}+w=0, \\
w=w_{x x}=0 \quad \text { at } \quad x=0, \pi .
\end{gathered}
$$

The eigenfunctions for this problem have the form

$$
w_{m}(x)=\sin m x, \quad m=1,2, \cdots
$$

and the corresponding eigenvalues are

$$
\lambda_{m}=\left[m^{2}+\left(1 / m^{2}\right)\right] / 2 .
$$

The classical buckling load (lowest eigenvalue) for this problem is $\lambda=1$ and the corresponding lateral deflection is $w=\sin x$. The length of the column is taken to be onehalf the wavelength of the classical buckling mode. The deflection of the column and the dynamic buckling load for small damping and imperfections and $\lambda$ close to 1 are sought.

Dynamic theory. The static theory has been studied in [8] and the dynamic theory with $\gamma=0$ has been studied in [6]. We consider $\epsilon<<1$ and wish to determine the maximum value $\lambda_{D}$ of $\lambda$ less than 1 such that the lateral deflection $w$ is bounded. As stated in [8], it is appropriate to use a perturbation in a load parameter for this problem. We set

$$
\delta^{2}=2(1-\lambda) .
$$

As in [6], it is also assumed that the solution depends on two time scales: $t$, which describes the short-time behavior of the solution and $\tau \equiv \delta t$, which describes the long-time behavior. We write $w(x, t) \equiv u(x, t, \tau ; \delta)$ and expand $u, \lambda \epsilon$ and $\gamma$ in power series in $\delta$ :

$$
\begin{gathered}
w(x, t) \equiv u(x, t, \tau ; \delta)=\sum_{n=1}^{\infty} u^{(n)}(x, t, \tau) \delta^{n}, \\
\lambda \epsilon=\sum_{n=1}^{\infty} \epsilon^{(n)} \delta^{n}, \quad \gamma=\sum_{n=1}^{\infty} \gamma^{(n)} \delta^{n} .
\end{gathered}
$$

The imperfection can be expanded in a Fourier sine series, i.e.

$$
\bar{w}(x)=\sum_{n=1}^{\infty} \dot{a}_{n} \sin n x .
$$

Substituting these expansions into (1) and equating like powers of $\delta$ yields the sequence of equations: 


$$
\begin{gathered}
L u^{(1)} \equiv u_{t t^{(1)}}+u_{x x x x}{ }^{(1)}+2 u_{x x}^{(1)}+u^{(1)}=-2 \epsilon^{(1)} \bar{w}_{x x} \\
L u^{(2)}=-2 \epsilon^{(2)} \bar{w}_{x x}-2 u_{t \tau}{ }^{(1)}-\gamma^{(1)} u_{t}^{(1)}, \\
L u^{(3)}=-2 \epsilon^{(3)} \bar{w}_{x x}-2 u_{t \tau}^{(2)}-\gamma^{(1)} u_{t}^{(2)}-\gamma^{(2)} u_{t}^{(1)}-u_{\tau \tau}{ }^{(1)} \\
+u_{x x}{ }^{(1)}-\gamma^{(1)} u_{\tau}^{(1)}+\alpha\left(u^{(1)}\right)^{3},
\end{gathered}
$$

etc. The boundary conditions (2) become

$$
u^{(j)}=u_{x x}^{(j)}=0 \quad \text { at } \quad x=0, \pi, t>0 ; \quad j=1,2, \cdots
$$

and the initial conditions (3) become

$$
\begin{gathered}
u^{(j)}(x, 0,0)=b^{(j)} f(x), \quad u_{t}^{(1)}(x, 0,0)=b^{(1)} g(x), \\
u_{t}^{(j+1)}(x, 0,0)+u_{\tau}^{(j)}(x, 0,0)=b^{(j+1)} g(x), \quad j=1,2, \cdots
\end{gathered}
$$

where

$$
b^{(j)}=\sum_{m=1}^{j} \epsilon^{(m)}\left[1+(-1)^{j-m}\right] 2^{(m-j-2) / 2}, \quad j=1,2, \cdots .
$$

As a preliminary step, necessary conditions for the existence of bounded solutions to (9) (13) are sought. Consider the problem

$$
\begin{aligned}
& L Y=h(x, t) \quad 0<x<\pi, t>0, \\
& Y=Y_{x x}=0 \quad \text { at } \quad x=0, \pi, t>0 .
\end{aligned}
$$

A solution of the form

$$
Y(x, t)=\sum_{n=1}^{\infty} Y_{n}(t) \sin n x
$$

exists if

$$
Y_{n}^{\prime \prime}+\left(n^{2}-1\right)^{2} Y_{n}=(2 / \pi) \int_{0}^{\pi} h(x, t) \sin n x d x
$$

In order to have solutions to this problem bounded in $t$, the inhomogeneous term must be orthogonal to the solutions of the corresponding homogeneous problem. Thus

$$
\begin{aligned}
& \lim _{T \rightarrow \infty}(1 / T) \int_{0}^{T} \int_{0}^{\pi} h(x, t) \sin n x \sin \left(n^{2}-1\right) t d x d t=0, \\
& \lim _{T \rightarrow \infty}(1 / T) \int_{0}^{T} \int_{0}^{\pi} h(x, t) \sin n x \cos \left(n^{2}-1\right) t d x d t=0 .
\end{aligned}
$$

Using the Fourier series expansion of the imperfection (8) and the orthogonality conditions (14) in Eq. (9) gives

$$
\epsilon^{(1)} \bar{a}_{1}=0 \text {. }
$$

According to [8], the imperfection in the shape of the classical buckling mode causes the greatest reduction in the strength of the column. Therefore, since we do not wish to neglect this term, we have $\dot{a}_{1} \neq 0$ and thus

$$
\epsilon^{(1)}=0
$$


Therefore, for $u^{(1)}$, Eqs. (9), (12) and (13) become

$$
\begin{gathered}
L u^{(1)}=0, \\
u^{(1)}=u_{x x}^{(1)}=0 \quad \text { at } \quad x=0, \pi, t>0, \\
u^{(1)}(x, 0,0)=u_{t}^{(1)}(x, 0,0)=0 .
\end{gathered}
$$

Let

$$
u^{(1)}(x, t, \tau)=\sum_{n=1}^{\infty} u_{n}^{(1)}(t, \tau) \sin n x
$$

Then

$$
u_{n, t t^{(1)}}+\left(n^{2}-1\right)^{2} u_{n}^{(1)}=0, \quad u_{n}^{(1)}(0,0)=u_{n, t}^{(1)}(0,0)=0, \quad n=1,2,
$$

If $n=1$, the bounded solution is

$$
u_{1}^{(1)}(t, \tau)=B_{1}^{(1)}(\tau)
$$

where

$$
B_{1}^{(1)}(0)=0 \text {. }
$$

If $n \neq 1$,

$$
u_{n}^{(1)}(t, \tau)=A_{n}^{(1)}(\tau) \sin \left(n^{2}-1\right) t+B_{n}^{(1)}(\tau) \cos \left(n^{2}-1\right) t
$$

where

$$
A_{n}^{(1)}(0)=B_{n}^{(1)}(0)=0 .
$$

Substitution of $u^{(1)}$ into (10) and the use of (8) and (14) yields

$$
\begin{aligned}
& \epsilon^{(2)}=0 ; \quad A_{n, \tau^{(1)}}+(1 / 2) \gamma^{(1)} A_{n}{ }^{(1)}=0, \\
& B_{n, \tau^{(1)}}+(1 / 2) \gamma^{(1)} B_{n}{ }^{(1)}=0 . \quad n \geq 2
\end{aligned}
$$

Use of the initial conditions for $A_{n}{ }^{(1)}$ and $B_{n}{ }^{(1)}$ gives $A_{n}{ }^{(1)}(\tau) \equiv 0, B_{n}{ }^{(1)}(\tau) \equiv 0$ for $n>1$, and thus

$$
u^{(1)}(x, t, \tau)=B_{1}^{(1)}(\tau) \sin x
$$

The equations for $u^{(2)}$ then become

$$
\begin{gathered}
L u^{(2)}=0, \quad 0<x<\pi, t>0, \\
u^{(2)}=u_{x x}^{(2)}=0 \quad \text { at } \quad x=0, \pi, t>0, \\
u^{(2)}(x, 0,0)=0, \\
u_{t}^{(2)}(x, 0,0)+u_{\tau}^{(1)}(x, 0,0)=0 .
\end{gathered}
$$

Following the same procedure used for $u^{(1)}$, i.e. letting

$$
u^{(2)}(x, t, \tau)=\sum_{n=1}^{\infty} u_{n}^{(2)}(t, \tau) \sin n x
$$

and using the boundedness conditions, we obtain

$$
u^{(2)}(x, t, \tau)=B_{1}^{(2)}(\tau) \sin x+\sum_{n=2}^{\infty}\left[A_{n}^{(2)}(\tau) \sin \left(n^{2}-1\right) t+B_{n}^{(2)}(\tau) \cos \left(n^{2}-1\right) t\right] \sin n x
$$


where $B_{1}^{(2)}(0)=0$ and $A_{n}^{(2)}(0)=B_{n}^{(2)}(0)=0$ for $n>1$. The boundedness condition also gives

$$
B_{1, \tau^{(1)}(0)}=0 .
$$

Using the results for $u^{(1)}$ and $u^{(2)}$ in (11) and applying the boundedness condition leads to

$$
\begin{gathered}
A_{n, \tau^{(2)}}+(1 / 2) \gamma^{(1)} A_{n}^{(2)}=0, \\
B_{n, \tau^{(2)}}+(1 / 2) \gamma^{(1)} B_{n}^{(2)}=0, \quad n \geq 2
\end{gathered}
$$

and

$$
B_{1, \tau \tau^{(1)}}+\gamma^{(1)} B_{1, \tau}{ }^{(1)}+B_{1}^{(1)}-(3 / 4) \alpha\left(B_{1}^{(1)}\right)^{3}=2 \epsilon^{(3)} \bar{a}_{1} .
$$

It is easily shown that $A_{n}{ }^{(2)}=B_{n}^{(2)} \equiv 0$ for $n \geq 2$. Since our primary interest is in obtaining information about $u^{(1)}$ and the dynamic buckling load, the computation of $u^{(2)}$ will not be considered further. To simplify the notation we let

$$
B(\tau) \equiv B_{1}^{(1)}(\tau),()^{\prime} \equiv d() / d \tau, c \equiv 2 \epsilon^{(3)} \bar{a}_{1}, \Gamma \equiv \gamma^{(1)} .
$$

Thus

$$
B^{\prime \prime}+\Gamma B^{\prime}+B-(3 / 4) \alpha B^{3}=c,
$$

where

$$
B(0)=B^{\prime}(0)=0 .
$$

If $\Gamma=0$, the problem is reduced to the one studied in [6]. In this case (17) can be multiplied by $B^{\prime}$ and integrated to obtain

$$
\left(B^{\prime}\right)^{2}+B^{2}-(3 / 8) \alpha B^{4}=2 c B
$$

This equation can be solved in terms of elliptic integrals, but a phase-plane analysis will be used to study the qualitative nature of the solution. This undamped case was studied in [6] and the results will be summarized here. All solutions to (18) and (19) are bounded and periodic if $\alpha=-1$. If $\alpha=1$, then (18) and (19) have bounded periodic solutions for all $\tau$ if $|c|<2 \sqrt{2} / 9$, have unbounded solutions as $\tau \rightarrow \infty$ if $|c|>2 \sqrt{2} / 9$ and have bounded solutions where $\left(B, B^{\prime}\right) \rightarrow(2 \sqrt{ } 2 / 3,0)$ as $\tau \rightarrow \infty$ if $|c|=2 \sqrt{2} / 9$.

If $\Gamma>0$, the problem is somewhat more difficult. Eq. (17) cannot be integrated in the same manner as (19); it is therefore necessary to reduce (17) to a system of two first-order equations:

$$
\begin{gathered}
B^{\prime}=V, \\
V^{\prime}=-\Gamma V-B+(3 / 4) \alpha B^{3}+c,
\end{gathered}
$$

where

$$
B(0)=V(0)=0
$$

We let

$$
\begin{gathered}
A^{+}=\left[(2 / 3 \alpha)\left(-c+\left(c^{2}-16 / 81 \alpha\right)^{1 / 2}\right)\right]^{1 / 3}, \\
A^{-}=\left[(2 / 3 \alpha)\left(-c-\left(c^{2}-16 / 81 \alpha\right)^{1 / 2}\right)\right]^{1 / 3}, \\
B_{1}=A^{+}+A^{-} .
\end{gathered}
$$


We first consider $\alpha=-1$. Then $B=B_{1}, V=0$ is the only singular point of (20). If $\Gamma>2[1$ $\left.+(9 / 4) B_{1}{ }^{2}\right]^{1 / 2}$ then $\left(B_{1}, 0\right)$ is a stable node; if $\Gamma<2\left[1+(9 / 4) B_{1}{ }^{2}\right]^{1 / 2}$ then $\left(B_{1}, 0\right)$ is a stable spiral point. We then find that our solutions are bounded as $\tau \rightarrow \infty$.

If $\alpha=1$ and $|c|>4 / 9$ then $\left(B_{1}, 0\right)$ is again the only singular point of (20). In this case, however, $\left(B_{1}, 0\right)$ is a saddle point and our solutions are unbounded as $\tau \rightarrow \infty$. If $\alpha=1$ and $|c|<4 / 9$, then there are three singular points of $(20)$ : at $\left(B_{1}, 0\right),\left(B_{2}, 0\right)$ and $\left(B_{3}, 0\right)$, where

$$
\begin{aligned}
& B_{2}=-(1 / 2)\left(A^{+}+A^{-}\right)+(i \sqrt{ } 3 / 2)\left(A^{+}-A^{-}\right), \\
& B_{3}=-(1 / 2)\left(A^{+}+A^{-}\right)-(i \sqrt{ } 3 / 2)\left(A^{+}-A^{-}\right) .
\end{aligned}
$$

Without loss of generality, let $B_{2}<B_{3}<B_{1}$. Then $\left(B_{1}, 0\right)$ and $\left(B_{2}, 0\right)$ are both saddle points. If $\Gamma>2\left[1-(9 / 4) B_{3}{ }^{2}\right]^{1 / 2}$ then $\left(B_{3}, 0\right)$ is a stable node; if $\Gamma<2\left[1-(9 / 4) B_{3}{ }^{2}\right]^{1 / 2}$ then $\left(B_{3}, 0\right)$ is a stable spiral point. If $c>0$, then $B_{2}<0<B_{3}$ and if $c<0$ then $B_{3}<0<B_{1}$. Thus, the initial point $(0,0)$ always lies between a saddle point and either a stable node or stable spiral point. For a given value of $|c|<4 / 9$, whether or not a curve in the phase plane passing through the origin will be "captured" by the stable singular point $\left(B_{3}, 0\right)$ as $\tau \rightarrow \infty$ depends on the value of $\Gamma$. We introduce a nondecreasing function $h$, defined for $\Gamma$ $\geq 0$. A numerical technique, similar to one found in [11], gives the following result: for each $\Gamma \geq 0$, if $|c|>h(\Gamma)$ then the solution is unbounded as $\tau \rightarrow \infty$ and if $|c|<h(\Gamma)$ then the solution is bounded. For all $\Gamma \geq 0,2 \sqrt{2} / 9 \leq h(\Gamma) \leq 4 / 9$, and for all $\Gamma \geq \Gamma^{*}, h(\Gamma)=4$ / 9. Thus far the numerical results indicate that $.95<\Gamma^{*}<1$. Table 1 gives $h$ to four significant digits.

We now summarize. If $\alpha=-1$, then the solutions to (17) and (18) are bounded if $\mathrm{\Gamma}>$ 0 and are bounded and periodic if $\Gamma=0$. If $\alpha=1$ then the solutions to (17) and (18) are bounded if $|c|<h(\Gamma)$ and unbounded as $\tau \rightarrow \infty$ if $|c|>h(\Gamma)$.

Using Eq. (6) and the fact that $\epsilon^{\prime 1}=\epsilon^{\prime 2}=0$ we find that

$$
\lambda \epsilon=\epsilon^{\prime 3} \delta^{3}+0\left(\delta^{4}\right) .
$$

Therefore

$$
c \sim[2(1-\lambda)]^{-3 / 2} 2 \lambda \epsilon \bar{a}_{1} \text { for } \lambda \rightarrow 1^{-} .
$$

That $c$ is a monotone increasing function of $\lambda$ for $0<\lambda<1$ is clear from (21). We define the dynamic buckling load $\lambda_{D}$ in the following way: for $0<\lambda<\lambda_{D}$ there are bounded solutions to (17) and (18), while for $\lambda_{D}<\lambda<1$ the solutions are unbounded. The use of

TABLE 1.

I $h(\Gamma)$

\begin{tabular}{c|c}
\hline 0.00 & $2,2 / 9 \sim .3143$ \\
0.10 & .3363 \\
0.20 & .3572 \\
0.30 & .3764 \\
0.40 & .3938 \\
0.50 & .4091 \\
0.60 & .4221 \\
0.70 & .4323 \\
0.80 & .4397 \\
0.90 & .4437 \\
$\geq 1.00$ & $4 / 9 \sim .4444$ \\
\hline
\end{tabular}


the critical value of $c$ for $\alpha=1$ in Eq. (21) gives a simple asymptotic expression for $\lambda_{D}$ :

$$
\left(1-\lambda_{D}\right)^{3 / 2}=(1 / \sqrt{ } 2 h(\Gamma)) \lambda_{D}\left|\epsilon \bar{a}_{1}\right|, \quad \Gamma \geq 0 .
$$

In [8] and elsewhere, the static buckling load is given asymptotically by

$$
\left(1-\lambda_{S}\right)^{3 / 2}=(9 / 4 \sqrt{ } 2) \lambda_{S}\left|\epsilon \tilde{a}_{1}\right| .
$$

Thus, as in [6]-[8], a simple relation between $\lambda_{D}$ and $\lambda_{S}$ may be obtained by eliminating $\left|\epsilon \bar{a}_{1}\right|$ from (22) and (23):

$$
\left[\left(1-\lambda_{D}\right) /\left(1-\lambda_{S}\right)\right]^{3 / 2}=(4 / 9)(1 / h(\Gamma)) \lambda_{D} / \lambda_{S}
$$

Note that if $h(\Gamma)=4 / 9$, i.e. if $\Gamma \geq \Gamma^{*}$, then $\lambda_{D}=\lambda_{S}$.

For $\Gamma=0, \lambda=\lambda_{D}$,

$$
B(\tau)=4 \sqrt{ } 2 /\left\{3\left[3 \operatorname{coth}^{2}(\tau / 2)-1\right]\right\}
$$

and

$$
w(x, t) \sim \frac{8\left(1-\lambda_{D}\right)^{1 / 2} \sin x}{3\left\{3 \operatorname{coth}^{2}\left[\left(1-\lambda_{D}\right)^{1 / 2} t / \sqrt{ } 2\right]-1\right\}} .
$$

Thus, under step-loading, the dynamic buckling load can be predicted from the static buckling load and the damping coefficient, independently of the small imperfection. If the initial displacement and velocity are restricted to be of the same order as the imperfection, then the dominant terms for the deflection and the dynamic buckling load depend only on the Fourier coefficient corresponding to imperfection in the shape of the classical buckling mode and on the damping. Asymptotically, the static and dynamic buckling loads are the same if the damping exceeds a fixed value.

\section{REFERENCES}

[1] W. T. Koiter, On the stability of elastic equilibrium (in Dutch), Thesis, Delft, Amsterdam (1945): English translation issued as NASA TT F-10, 1967

[2] W. T. Koiter, Elastic stability and post-buckling behavior, in Nonlinear problems, ed. R. E. Langer. University of Wisconsin Press, Madison, 1963

[3] B. Budiansky and J. W. Hutchinson, Dynamic buckling of imperfection-sensitive structures, in Proceedings of the Eleventh International Congress of A pplied Mechanics, ed. H. Gortler, Springer-Verlag, 1966, 636-651

[4] J. W. Hutchinson and B. Budiansky, Dynamic buckling estimates, A.I.A.A. J. 4, 525-530 (1966)

[5] B. Budiansky, Dynamic buckling of elastic structures: criteria and estimates, in Dynamic stability of structures, ed. G. Herrmann, Pergamon, New York, 1966

[6] J. C. A mazigo and D. Frank, Dynamic buckling of an imperfect column on nonlinear foundation, Quart. Appl. Math. 31, 1-9 (1973)

[7] W. B. Fraser and B. Budiansky, The buckling of a column with random initial deflections, J. Appl. Mech. 36. 233-240 (1969)

[8] J. C. A mazigo, B. Budiansky and G. F. Carrier, Asymptotic analyses of the buckling of imperfect columns on nonlinear elastic foundations, Int. J. Solids. Struct. 6, 1341-1356 (1970)

[9] J. C. Amazigo, Buckling of stochastically imperfect columns on nonlinear elastic foundations, Quart. Appl. Math. 29, 403-409 (1971)

[10] E. L. Reiss and B. J. Matkowsky, Nonlinear dynamic buckling of a compressed elastic column, Quart. Appl. Math. 29, 245-260 (1971)

[11] J. J. Stoker, Nonlinear vibrations in mechanical and electrical systems, Interscience, New York, 1950, 75-80 